1 Hacettepe Journal of Mathematics and Statistics

$\bigcap$ Volume 47 (6) (2018), $1427-1437$

\title{
On computation of some distance-based topological indices of circulant networks
}

\author{
Faisal Ali*, Abdul Hafeez ${ }^{\dagger}$, Muhammad Salman ${ }^{\ddagger}$ and Shuliang Huang ${ }^{\S}$
}

\begin{abstract}
The distance, $d(u, v)$, between two vertices $u$ and $v$ of a connected graph $G$ is the length of a $u-v$ geodesic in $G$. A large number of graph-distance-based topological indices in various families of graphs and networks have been computed. In this paper, we consider circulant networks and compute three distance-based topological indices, namely the Wiener index, hyper-Wiener index and Schultz molecular topological index on these networks.
\end{abstract}

Keywords: Wiener index, hyper-Wiener index and Schultz index, circulant network.

Mathematics Subject Classification (2010): $05 \mathrm{C} 12$

Received : 04.12.2015 Accepted : 07.01.2016 Doi: 10.15672/HJMS.2017.407

\section{Preliminaries}

A combination of chemistry, mathematics and information defines a new subject called cheminformatics. To predict the biological activities and properties of chemical compounds, cheminformatics studies Quantitative structure-activity and Quantitative structure-property relationships (QSAR/QSPR). In QSAR/QSPR study, physicochemical properties and topological indices such as Wiener index, hyper-Wiener index, Harary index, Randić index, Zagreb index and Schultz index are used to predict bioactivity of chemical compounds.

\footnotetext{
${ }^{*}$ Centre for Advanced Studies in Pure and Applied Mathematics, Bahauddin Zakariya University Multan, Pakistan, Email: faisalali@bzu.edu.pk

${ }^{\dagger}$ Centre for Advanced Studies in Pure and Applied Mathematics, Bahauddin Zakariya University Multan, Pakistan, Email: hafeez276@gmail.com

$\ddagger$ Department of Mathematics, Government Post Graduate College Bahawalpur, Pakistan Email: solo33@gmail.com

$\S$ Department of Mathematics, Chuzhou University, Chuzhou, China, Email: shulianghuang@163.com

ICorresponding Author.
} 
Let $\mathcal{G}$ be a family of graphs. A topological index is a function $T o p: \mathcal{G} \rightarrow \mathbb{R}$ such that $G \cong H$ implies $\operatorname{Top}(G)=\operatorname{Top}(H)$ for two graphs $G, H \in \mathcal{G}$ [8]. A topological index is, in fact, a numeric quantity associated with chemical constitution purporting for correlation of chemical structure with many physico-chemical properties. Actually, they are designed on the ground of transformation of a molecular graph into a number which characterizes the topology of that graph. Due to the chemical significance of topological indices, remarkable research has been done on topological indices of various families of graphs.

In this paper, we consider simple connected graphs $G$ with vertex set $V(G)$ and edge set $E(G)$. The number $d(v)$ denotes the degree of a vertex $v$ in $G$, which is the number of edges adjacent with $v$ in $G$. The number $d(u, v)$ denotes the distance between two vertices $u$ and $v$ of $G$, which is defined as the length of a shortest path between $u$ and $v$ in $G$. A network is simply a connected graph having no multiple edges. Here, we consider the family of circulant networks, which is defined as follows: Let $n, m$ and $a_{1}, a_{2}, \ldots, a_{m}$ be positive integers, $1 \leq a_{i} \leq\left\lfloor\frac{n}{2}\right\rfloor$ and $a_{i} \neq a_{j}$ for all $1 \leq i<j \leq m$. An undirected graph with the set of vertices $\left\{v_{i+1} ; i \in Z_{n}\right\}\left(Z_{n}\right.$ : the additive group of integers modulo $\left.n\right)$ and the set of edges $\left\{v_{j} v_{j+a_{l}}: 1 \leq j \leq n, 1 \leq l \leq m\right\}$ is called a circulant graph, and is denoted by $C_{n}\left(a_{1}, a_{2}, \ldots, a_{m}\right)$. The numbers $a_{1}, a_{2}, \ldots, a_{m}$ are called the generators and we say that the edge $v_{j} v_{j+a_{l}}$ is of type $a_{l}$. The indices after $n$ will be taken modulo $n$. It is easy to see that a circulant network $C_{n}\left(a_{1}, a_{2}, \ldots, a_{m}\right)$ is a regular graph of degree $r$, where

$$
r= \begin{cases}2 m-1 & \text { if } \frac{n}{2} \in\left\{a_{1}, a_{2}, \ldots, a_{m}\right\} \\ 2 m & \text { otherwise. }\end{cases}
$$

The class of circulant networks is an important class of graphs, which is useful in the design of local area networks [1]. Circulant networks have played a vital role for decades in the design of computer and telecommunication networks due to their optimal faulttolerance and routing capabilities [2]. They also constitute the basis for designing certain data alignment networks for complex memory systems [14]. We consider two families of circulant graphs, $C_{n}(1, a)$ for $a=2,3$ in this paper, and compute the Wiener, hyperWiener and Schultz indices on these families, which are defined as follows:

In 1947, to study the boiling points of paraffins, Wiener introduced the first non-trivial distance-based topological index. He named this index the path number, and later on it was called the Wiener index [13]. The research interest in Wiener index and related indices is still considerable (see the bibliography and therein [9, 11]). This index was given in terms of edge weights which, originally, was defined on trees. Traditionally, its generalization on general graphs $G$ is defined as:

$$
W(G)=\sum_{u, v \in V(G)} d(u, v)
$$

During the last two decades, a large number of generalizations and extensions of the Wiener index has been introduced and studied by various mathematical chemists. An extensive bibliography on this matter can by viewed in $[4,7]$. One of these extensions, the hyper-Wiener index was proposed by Randić for trees [10], and extended to all connected graphs by Klein et al. [6]. This index remarkably used as a structure descriptor for predicting physicochemical properties of chemical compounds, which are significant for pharmacology, agriculture and environment protection $[3,6,10]$. This index is defined as:

$$
W W(G)=\frac{1}{2} \sum_{u, v \in V(G)} d(u, v)(1+d(u, v)) .
$$


Another generalization of the Wiener index is the Schultz molecular topological index, which was introduced in 1989 [12], and is defined as:

$$
\operatorname{MTI}(G)=\sum_{u \in V(G)} \sum_{v \in V(G)} d(u)\left(A_{u v}+d(u, v)\right)
$$

where $A_{u v}$ is the $(u, v)$-th entry of the adjacency matrix $A$ of $G$.

\section{Circulant networks $C_{n}(1,2)$}

In this section, we consider circulant networks $C_{n}(1,2)$, for all $n \geq 5$, in the context of the Wiener, hyper-Wiener and Schultz indices. Firstly, we define some notations which will be useful in the sequel. Let

$$
D(v \mid G)=\sum_{u \in V(G)} d(u, v) \quad \text { and } \quad D D(v \mid G)=\frac{1}{2}\left(D(v \mid G)+\sum_{u \in V(G)}(d(u, v))^{2}\right),
$$

and we call them the distance number and the double distance number of $v$, respectively. Then the Wiener and the hyper-Wiener indices can be expressed as:

$$
\begin{aligned}
W(G) & =\frac{1}{2} \sum_{v \in V(G)} D(v \mid G), \\
W W(G) & =\frac{1}{2} \sum_{v \in V(G)} D D(v \mid G) .
\end{aligned}
$$

The Schultz molecular topological index can also be expressed as [5]:

$$
\operatorname{MTI}(G)=\sum_{v \in V(G)}(d(v))^{2}+\sum_{v \in V(G)} d(v) D(v \mid G) .
$$

2.1. Theorem. For $n \geq 5$, let $G$ be a circulant network $C_{n}(1,2)$. Then

$W(G)=\frac{1}{16}\left\{\begin{array}{cl}n^{2}(n+2) & , \quad \text { when } n \equiv 0,2(\bmod 4), \\ n(n-1)(n+3) & , \quad \text { when } n \equiv 1(\bmod 4), \\ n(n+1)^{2} & , \quad \text { when } n \equiv 3(\bmod 4) .\end{array}\right.$

Proof. We discuss the following three cases:

Case 1: When $n \equiv 0,2(\bmod 4)$. For all $v \in V(G)$, the distance number of $v$ is

$$
\begin{aligned}
D(v \mid G) & =\left\{\begin{array}{c}
4\left(1+2+\ldots+\frac{n-4}{4}\right)+3\left(\frac{n}{4}\right) \\
4\left(1+2+\ldots+\frac{n-2}{4}\right)+1\left(\frac{n-2}{4}+1\right)
\end{array} \quad, \quad \text { when } n \equiv 0(\bmod 4),\right.
\end{aligned}
$$

By applying the formula of Wiener index, we have

$$
W(G)=\frac{1}{2} \sum_{v \in V(G)} D(v \mid G)=\frac{1}{2} \sum_{v \in V(G)} \frac{1}{8} n(n+2)=\frac{1}{16} n^{2}(n+2) .
$$

Case 2: When $n \equiv 1(\bmod 4)$. The distance number of each $v \in V(G)$ is

$$
D(v \mid G)=4\left(1+2+\ldots+\frac{n-1}{4}\right)=\frac{1}{8}(n-1)(n+3) .
$$

By applying the formula of Wiener index, we have 
$W(G)=\frac{1}{2} \sum_{v \in V(G)} D(v \mid G)=\frac{1}{2} \sum_{v \in V(G)} \frac{1}{8}(n-1)(n+3)=\frac{1}{16} n(n-1)(n+3)$.

Case 3: When $n \equiv 3(\bmod 4)$. The distance number of every vertex $v$ of $G$ is

$D(v \mid G)=4\left(1+2+\ldots+\frac{n-3}{4}\right)+2\left(\frac{n-3}{4}+1\right)=\frac{1}{8}(n+1)^{2}$.

By applying the formula of Wiener index, we have

$W(G)=\frac{1}{2} \sum_{v \in V(G)} D(v \mid G)=\frac{1}{2} \sum_{v \in V(G)} \frac{1}{8}(n+1)^{2}=\frac{1}{16} n(n+1)^{2}$.

2.2. Theorem. For $n \geq 5$, let $G$ be a circulant network $C_{n}(1,2)$. Then

$W W(G)=\frac{1}{192}\left\{\begin{array}{cl}n^{2}\left(n^{2}+9 n+20\right) & , \quad \text { when } n \equiv 0(\bmod 4), \\ n(n-1)(n+3)(n+7) & , \quad \text { when } n \equiv 1(\bmod 4), \\ n(n+2)\left(n^{2}+7 n+6\right) & , \quad \text { when } n \equiv 2(\bmod 4), \\ n(n+1)\left(n^{2}+8 n+15\right) & , \quad \text { when } n \equiv 3(\bmod 4) .\end{array}\right.$

Proof. We discuss the following four cases:

Case 1: When $n \equiv 0(\bmod 4)$. For all $v \in V(G)$, the double distance number of $v$ is

$D D(v \mid G)=\frac{1}{2}\left(D(v \mid G)+4\left(1^{2}+2^{2}+3^{2}+\ldots+\left(\frac{n-4}{4}\right)^{2}\right)+3\left(\frac{n}{4}\right)^{2}\right)$.

Using the value of $D(v \mid G)$ derived in Theorem 2.1 (Case-1), we have

$D D(v \mid G)=\frac{1}{96} n\left(n^{2}+9 n+20\right)$.

By applying the formula of hyper-Wiener index, we have

$$
\begin{aligned}
W W(G) & =\frac{1}{2} \sum_{v \in V(G)} D D(v \mid G) \\
& =\frac{1}{2} \sum_{v \in V(G)} \frac{1}{96} n\left(n^{2}+9 n+20\right)=\frac{1}{192} n^{2}\left(n^{2}+9 n+20\right) .
\end{aligned}
$$

Case 2: When $n \equiv 1(\bmod 4)$. For all $v \in V(G)$, the double distance number of $v$ is

$D D(v \mid G)=\frac{1}{2}\left(D(v \mid G)+4\left(1^{2}+2^{2}+3^{2}+\ldots+\left(\frac{n-1}{4}\right)^{2}\right)\right)$.

Using the value of $D(v \mid G)$ derived in Theorem 2.1 (Case-1), we have

$D D(v \mid G)=\frac{1}{96}(n-1)(n+3)(n+7)$.

By applying the formula of hyper-Wiener index, we have 


$$
\begin{aligned}
W W(G) & =\frac{1}{2} \sum_{v \in V(G)} D D(v \mid G) \\
& =\frac{1}{2} \sum_{v \in V(G)} \frac{1}{96}(n-1)(n+3)(n+7)=\frac{1}{192} n(n-1)(n+3)(n+7) .
\end{aligned}
$$

Case 3: When $n \equiv 2(\bmod 4)$. For each vertex $v$ of $G$, the double distance number of $v$ is

$$
D D(v \mid G)=\frac{1}{2}\left(D(v \mid G)+4\left(1^{2}+2^{2}+3^{2}+\ldots+\left(\frac{n-2}{4}\right)^{2}\right)+1\left(\frac{n-2}{4}+1\right)^{2}\right) .
$$

Using the value of $D(v \mid G)$ derived in Theorem 2.1 (Case-2), we have

$D D(v \mid G)=\frac{1}{96}(n+2)\left(n^{2}+7 n+6\right)$.

By applying the formula of hyper-Wiener index, we have

$$
\begin{aligned}
W W(G) & =\frac{1}{2} \sum_{v \in V(G)} D D(v \mid G) \\
& =\frac{1}{2} \sum_{v \in V(G)} \frac{1}{96}(n+2)\left(n^{2}+7 n+6\right)=\frac{1}{192} n(n+2)\left(n^{2}+7 n+6\right) .
\end{aligned}
$$

Case 4: When $n \equiv 3(\bmod 4)$. For all $v \in V(G)$, the double distance number of $v$ is

$D D(v \mid G)=\frac{1}{2}\left(D(v \mid G)+4\left(1^{2}+2^{2}+3^{2}+\ldots+\left(\frac{n-3}{4}\right)^{2}\right)+2\left(\frac{n-3}{4}+1\right)^{2}\right)$.

Using the value of $D(v \mid G)$ derived in Theorem 2.1 (Case-3), we have

$D D(v \mid G)=\frac{1}{96}(n+1)\left(n^{2}+8 n+15\right)$.

By applying the formula of hyper-Wiener index, we have

$$
\begin{aligned}
W W(G) & =\frac{1}{2} \sum_{v \in V(G)} D D(v \mid G) \\
& =\frac{1}{2} \sum_{v \in V(G)} \frac{1}{96}(n+1)\left(n^{2}+8 n+15\right)=\frac{1}{192} n(n+1)\left(n^{2}+8 n+15\right) .
\end{aligned}
$$

2.3. Theorem. For $n \geq 5$, let $G$ be a circulant network $C_{n}(1,2)$. Then

$$
\operatorname{MTI}(G)=\frac{1}{2} n \begin{cases}\left(n^{2}+2 n+32\right) & , \quad \text { when } n \equiv 0,2(\bmod 4), \\ \left(n^{2}+2 n+29\right) & , \quad \text { when } n \equiv 1(\bmod 4), \\ \left(n^{2}+2 n+33\right) & , \quad \text { when } n \equiv 3(\bmod 4)\end{cases}
$$

Proof. We discuss the following three cases:

Case 1: When $n \equiv 0,2(\bmod 4)$. Since the degree $d(v)$ of each vertex $v$ in $G$ is 4 , so using the distance number $D(v \mid G)$ for each $v \in V(G)$, derived in Theorem 2.1 (Case-1), and by applying the formula of Schultz molecular topological index, we have 


$$
\begin{aligned}
\operatorname{MTI}(G) & =\sum_{v \in V(G)}(d(v))^{2}+\sum_{v \in V(G)} d(v) D(v \mid G) \\
& =16 n+4 n\left(\frac{1}{8} n(n+2)\right)=\frac{1}{2} n\left(n^{2}+2 n+32\right) .
\end{aligned}
$$

Case 2: When $n \equiv 1(\bmod 4)$. Since the degree $d(v)$ of each vertex $v$ in $G$ is 4 , so using the distance number $D(v \mid G)$ for each $v \in V(G)$, derived in Theorem 2.1 (Case-2), and by applying the formula of Schultz molecular topological index, we have

$$
\begin{aligned}
\operatorname{MTI}(G) & =\sum_{v \in V(G)}(d(v))^{2}+\sum_{v \in V(G)} d(v) D(v \mid G) \\
& =16 n+4 n\left(\frac{1}{8}(n-1)(n+3)\right)=\frac{1}{2} n\left(n^{2}+2 n+29\right) .
\end{aligned}
$$

Case 3: When $n \equiv 3(\bmod 4)$. Since the degree $d(v)$ of each vertex $v$ in $G$ is 4 , so using the distance number $D(v \mid G)$ for each $v \in V(G)$, derived in Theorem 2.1 (Case-3), and by applying the formula of Schultz molecular topological index, we have

$$
\begin{aligned}
\operatorname{MTI}(G) & =\sum_{v \in V(G)}(d(v))^{2}+\sum_{v \in V(G)} d(v) D(v \mid G) \\
& =16 n+4 n\left(\frac{1}{8}(n+1)^{2}\right)=\frac{1}{2} n\left(n^{2}+2 n+33\right) .
\end{aligned}
$$

\section{Circulant networks $C_{n}(1,3)$}

In this section, we consider circulant networks $C_{n}(1,3)$, for all $n \geq 7$, in the context of the Wiener, hyper-Wiener and Schultz indices.

3.1. Theorem. For $n \geq 7$, let $G$ be a circulant network $C_{n}(1,3)$. Then

$W(G)=\frac{1}{24}\left\{\begin{array}{cl}n^{2}(n+8) & , \text { when } n \equiv 0,4(\bmod 6), \\ n(n+9)(n-1) & , \text { when } n \equiv 1,3(\bmod 6), \\ n\left(n^{2}+8 n-8\right) & , \quad \text { when } n \equiv 2(\bmod 6), \\ n\left(n^{2}+8 n+7\right) & , \quad \text { when } n \equiv 5(\bmod 6) .\end{array}\right.$

Proof. We discuss the following four cases:

Case 1: When $n \equiv 0,4(\bmod 6)$. For all $v \in V(G)$, the distance number of $v$ is

$$
\begin{aligned}
D(v \mid G) & =\left\{\begin{array}{cc}
4(1)+6\left(2+3+\ldots+\frac{n-6}{6}\right)+5\left(\frac{n}{6}\right)+2\left(\frac{n+6}{6}\right) & , \quad n \equiv 0(\bmod 6), \\
4\left(1+\frac{n+2}{6}\right)+6\left(2+3+\ldots+\frac{n-4}{6}\right)+1\left(\frac{n+8}{6}\right) & , \quad n \equiv 4(\bmod 6),
\end{array}\right. \\
& =\frac{1}{12} n(n+8) .
\end{aligned}
$$

By applying the formula of Wiener index, we have

$$
W(G)=\frac{1}{2} \sum_{v \in V(G)} D(v \mid G)=\frac{1}{2} \sum_{v \in V(G)} \frac{1}{12} n(n+8)=\frac{1}{24} n^{2}(n+8) .
$$

Case 2: When $n \equiv 1,3(\bmod 6)$. For all $v \in V(G)$, the distance number of $v$ is 


$$
\begin{aligned}
& D(v \mid G)=\left\{\begin{array}{cll}
\left.4(1)+6\left(2+3+\ldots+\frac{n-1}{6}\right)+2\left(\frac{n+5}{6}\right)\right) & , & \text { when } n \equiv 1(\bmod 6), \\
4\left(1+\frac{n+3}{6}\right)+6\left(2+3+\ldots+\frac{n-3}{6}\right) & , & \text { when } n \equiv 3(\bmod 6),
\end{array}\right. \\
& =\frac{1}{12}(n+9)(n-1) \text {. }
\end{aligned}
$$

By applying the formula of Wiener index, we have

$$
W(G)=\frac{1}{2} \sum_{v \in V(G)} D(v \mid G)=\frac{1}{2} \sum_{v \in V(G)} \frac{1}{12}(n+9)(n-1)=\frac{1}{24} n(n+9)(n-1) .
$$

Case 3: When $n \equiv 2(\bmod 6)$. For all $v \in V(G)$, the distance number of $v$ is

$$
\begin{aligned}
D(v \mid G) & =4(1)+6\left(2+3+\ldots+\frac{n-2}{6}\right)+3\left(\frac{n+4}{6}\right) \\
& =\frac{1}{12}\left(n^{2}+8 n-8\right) .
\end{aligned}
$$

By applying the formula of Wiener index, we have

$$
W(G)=\frac{1}{2} \sum_{v \in V(G)} D(v \mid G)=\frac{1}{2} \sum_{v \in V(G)} \frac{1}{12}\left(n^{2}+8 n-8\right)=\frac{1}{24} n\left(n^{2}+8 n-8\right) .
$$

Case 4: When $n \equiv 5(\bmod 6)$. For all $v \in V(G)$, the distance number of $v$ is

$$
\begin{aligned}
D(v \mid G) & =4\left(1+\frac{n+1}{6}\right)+6\left(2+3+\ldots+\frac{n-5}{6}\right)+2\left(\frac{n+7}{6}\right) \\
& =\frac{1}{12}\left(n^{2}+8 n+7\right) .
\end{aligned}
$$

By applying the formula of Wiener index, we have

$W(G)=\frac{1}{2} \sum_{v \in V(G)} D(v \mid G)=\frac{1}{2} \sum_{v \in V(G)} \frac{1}{12}\left(n^{2}+8 n+7\right)=\frac{1}{24} n\left(n^{2}+8 n+7\right)$.

3.2. Theorem. For $n \geq 7$, let $G$ be a circulant network $C_{n}(1,3)$. Then

$$
W W(G)=\frac{1}{432}\left\{\begin{array}{cl}
n^{2}\left(n^{2}+21 n+162\right) & , \quad \text { when } n \equiv 0(\bmod 6), \\
n\left(n^{3}+21 n^{2}+135 n-157\right) & , \text { when } n \equiv 1(\bmod 6), \\
n\left(n^{3}+12 n^{2}+138 n-152\right) & , \text { when } n \equiv 2(\bmod 6), \\
n\left(n^{3}+21 n^{2}+135 n-189\right) & , \text { when } n \equiv 3(\bmod 6), \\
n\left(n^{3}+21 n^{2}+162 n+32\right) & , \text { when } n \equiv 4(\bmod 6), \\
n\left(n^{3}+21 n^{2}+183 n+163\right) & , \quad \text { when } n \equiv 5(\bmod 6) .
\end{array}\right.
$$

Proof. We discuss the following six cases:

Case 1: When $n \equiv 0(\bmod 6)$. For all $v \in V(G)$, the double distance number of $v$ is

$$
D D(v \mid G)=\frac{1}{2}\left(D(v \mid G)+4\left(1^{2}\right)+6\left(2^{2}+\ldots+\left(\frac{n-6}{6}\right)^{2}\right)+5\left(\frac{n}{6}\right)^{2}+2\left(\frac{n+6}{6}\right)^{2}\right) .
$$

Using the value of $D(v \mid G)$ derived in Theorem 3.1 (Case-1), we have

$$
D D(v \mid G)=\frac{1}{216} n\left(n^{2}+21 n+162\right) .
$$


By applying the formula of hyper-Wiener index, we have

$$
\begin{aligned}
W W(G) & =\frac{1}{2} \sum_{v \in V(G)} D D(v \mid G) \\
& =\frac{1}{2} \sum_{v \in V(G)} \frac{1}{216} n\left(n^{2}+21 n+162\right)=\frac{1}{432} n^{2}\left(n^{2}+21 n+162\right) .
\end{aligned}
$$

Case 2: When $n \equiv 1(\bmod 6)$. For all $v \in V(G)$, the double distance number of $v$ is $D D(v \mid G)=\frac{1}{2}\left(D(v \mid G)+4\left(1^{2}\right)+6\left(2^{2}+3^{2}+\ldots+\left(\frac{n-1}{6}\right)^{2}+2\left(\frac{n+5}{6}\right)^{2}\right)\right)$.

Using the value of $D(v \mid G)$ derived in Theorem 3.1 (Case-2), we have $D D(v \mid G)=\frac{1}{216}\left(n^{3}+21 n^{2}+135 n-157\right)$.

By applying the formula of hyper-Wiener index, we have

$$
\begin{aligned}
W W(G) & =\frac{1}{2} \sum_{v \in V(G)} D D(v \mid G) \\
& =\frac{1}{2} \sum_{v \in V(G)} \frac{1}{216}\left(n^{3}+21 n^{2}+135 n-157\right) \\
& =\frac{1}{432} n\left(n^{3}+21 n^{2}+135 n-157\right) .
\end{aligned}
$$

Case 3: When $n \equiv 2(\bmod 6)$. For all $v \in V(G)$, the double distance number of $v$ is $D D(v \mid G)=\frac{1}{2}\left(D(v \mid G)+4\left(1^{2}\right)+6\left(2^{2}+3^{2}+\ldots+\left(\frac{n-2}{6}\right)^{2}\right)+3\left(\frac{n+4}{6}\right)^{2}\right)$.

Using the value of $D(v \mid G)$ derived in Theorem 3.1 (Case-3), we have $D D(v \mid G)=\frac{1}{216}\left(n^{3}+21 n^{2}+138 n-152\right)$.

By applying the formula of hyper-Wiener index, we have

$$
\begin{aligned}
W W(G) & =\frac{1}{2} \sum_{v \in V(G)} D D(v \mid G) \\
& =\frac{1}{2} \sum_{v \in V(G)} \frac{1}{216}\left(n^{3}+21 n^{2}+138 n-152\right) \\
& =\frac{1}{432} n\left(n^{3}+21 n^{2}+138 n-152\right) .
\end{aligned}
$$

Case 4: When $n \equiv 3(\bmod 6)$. For all $v \in V(G)$, the double distance number of $v$ is $D D(v \mid G)=\frac{1}{2}\left(D(v \mid G)+4\left(1^{2}+\left(\frac{n+3}{6}\right)^{2}\right)+6\left(2^{2}+3^{2}+\ldots+\left(\frac{n-3}{6}\right)^{2}\right)\right)$.

Using the value of $D(v \mid G)$ derived in Theorem 3.1 (Case-2), we have 
$D D(v \mid G)=\frac{1}{216}\left(n^{3}+21 n^{2}+135 n-189\right)$.

By applying the formula of hyper-Wiener index, we have

$$
\begin{aligned}
W W(G) & =\frac{1}{2} \sum_{v \in V(G)} D D(v \mid G) \\
& =\frac{1}{2} \sum_{v \in V(G)} \frac{1}{216}\left(n^{3}+21 n^{2}+135 n-189\right) \\
& =\frac{1}{432} n\left(n^{3}+21 n^{2}+135 n-189\right) .
\end{aligned}
$$

Case 5: When $n \equiv 4(\bmod 6)$. For all $v \in V(G)$, the double distance number of $v$ is

$$
D D(v \mid G)=\frac{1}{2}\left(D(v \mid G)+4\left(1^{2}+\left(\frac{n+2}{6}\right)^{2}\right)+6\left(2^{2}+\ldots+\left(\frac{n-4}{6}\right)^{2}\right)+1\left(\frac{n+8}{6}\right)^{2}\right) .
$$

Using the value of $D(v \mid G)$ derived in Theorem 3.1 (Case-1), we have

$$
D D(v \mid G)=\frac{1}{216}\left(n^{3}+21 n^{2}+162 n+32\right) .
$$

By applying the formula of hyper-Wiener index, we have

$$
\begin{aligned}
W W(G) & =\frac{1}{2} \sum_{v \in V(G)} D D(v \mid G) \\
& =\frac{1}{2} \sum_{v \in V(G)} \frac{1}{216}\left(n^{3}+21 n^{2}+162 n+32\right) \\
& =\frac{1}{432} n\left(n^{3}+21 n^{2}+162 n+32\right) .
\end{aligned}
$$

Case 6: When $n \equiv 5(\bmod 6)$. For all $v \in V(G)$, the double distance number of $v$ is

$$
D D(v \mid G)=\frac{1}{2}\left(D(v \mid G)+4\left(1^{2}+\left(\frac{n+1}{6}\right)^{2}\right)+6\left(2^{2}+\ldots+\left(\frac{n-5}{6}\right)^{2}\right)+2\left(\frac{n+7}{6}\right)^{2}\right) .
$$

Using the value of $D(v \mid G)$ derived in Theorem 3.1 (Case-4), we have

$$
D D(v \mid G)=\frac{1}{216}\left(n^{3}+21 n^{2}+183 n+163\right) .
$$

By applying the formula of hyper-Wiener index, we have

$$
\begin{aligned}
W W(G) & =\frac{1}{2} \sum_{v \in V(G)} D D(v \mid G) \\
& =\frac{1}{2} \sum_{v \in V(G)} \frac{1}{216}\left(n^{3}+21 n^{2}+183 n+163\right) \\
& =\frac{1}{432} n\left(n^{3}+21 n^{2}+183 n+163\right) .
\end{aligned}
$$


3.3. Theorem. For $n \geq 7$, let $G$ be a circulant network $C_{n}(1,3)$. Then

$$
\operatorname{MTI}(G)=\frac{1}{3} n \begin{cases}\left(n^{2}+8 n+48\right) & , \quad \text { when } n \equiv 0,4(\bmod 6), \\ \left(n^{2}+8 n+39\right) & , \text { when } n \equiv 1,3(\bmod 6), \\ \left(n^{2}+8 n+40\right) & , \text { when } n \equiv 2(\bmod 6), \\ \left(n^{2}+8 n+55\right) & , \quad \text { when } n \equiv 5(\bmod 6) .\end{cases}
$$

Proof. We discuss the following four cases:

Case 1: When $n \equiv 0,4(\bmod 6)$. Since the degree $d(v)$ of each vertex $v$ in $G$ is 4 , so using the distance number $D(v \mid G)$ for each $v \in V(G)$, derived in Theorem 3.1 (Case-1), and by applying the formula of Schultz molecular topological index, we have

$$
\begin{aligned}
\operatorname{MTI}(G) & =\sum_{v \in V(G)}(d(v))^{2}+\sum_{v \in V(G)} d(v) D(v \mid G) \\
& =16 n+4 n\left(\frac{1}{12} n(n+8)\right)=\frac{1}{3} n\left(n^{2}+8 n+48\right) .
\end{aligned}
$$

Case 2: When $n \equiv 1,3(\bmod 6)$. Since the degree $d(v)$ of each vertex $v$ in $G$ is 4 , so using the distance number $D(v \mid G)$ for each $v \in V(G)$, derived in Theorem 3.1 (Case-2), and by applying the formula of Schultz molecular topological index, we have

$$
\begin{aligned}
\operatorname{MTI}(G) & =\sum_{v \in V(G)}(d(v))^{2}+\sum_{v \in V(G)} d(v) D(v \mid G) \\
& =16 n+4 n\left(\frac{1}{12} n\left(n^{2}+8 n+3\right)\right)=\frac{1}{3} n\left(n^{2}+8 n+39\right) .
\end{aligned}
$$

Case 3: When $n \equiv 2(\bmod 6)$. Since the degree $d(v)$ of each vertex $v$ in $G$ is 4 , so using the distance number $D(v \mid G)$ for each $v \in V(G)$, derived in Theorem 3.1 (Case-3), and by applying the formula of Schultz molecular topological index, we have

$$
\begin{aligned}
\operatorname{MTI}(G) & =\sum_{v \in V(G)}(d(v))^{2}+\sum_{v \in V(G)} d(v) D(v \mid G) \\
& =16 n+4 n\left(\frac{1}{12}\left(n^{2}+8 n-8\right)\right)=\frac{1}{3} n\left(n^{2}+8 n+40\right) .
\end{aligned}
$$

Case 4: When $n \equiv 5(\bmod 6)$. Since the degree $d(v)$ of each vertex $v$ in $G$ is 4 , so using the distance number $D(v \mid G)$ for each $v \in V(G)$, derived in Theorem 3.1 (Case-4), and by applying the formula of Schultz molecular topological index, we have

$$
\begin{aligned}
\operatorname{MTI}(G) & =\sum_{v \in V(G)}(d(v))^{2}+\sum_{v \in V(G)} d(v) D(v \mid G) \\
& =16 n+4 n\left(\frac{1}{12}\left(n^{2}+8 n+7\right)\right)=\frac{1}{3} n\left(n^{2}+8 n+55\right) .
\end{aligned}
$$

Acknowledgment. This research work was supported by the Anhui Provincial Natural Science Foundation (1408085QA08) and the Key University Science Research Project of Anhui Province (KJ2014A183) and also the Training Program of Chuzhou University (2014PY06) of China. 


\section{References}

[1] Bermound, J.C., Comellas, F. and Hsu, D. F. Distributed loop computer networks: survey, J. Parallel Distrib. Comput. 24, 2-10, 1995.

[2] Boesch, F.T. and Wang, J. Reliable circulant networks with minimum transmission delay, IEEE Transactions on Circuit and Systems 32, 1286-1291, 1985.

[3] Devillers, J. and Balaban, A.T. (Eds.) Topological indices and related descriptors in QSAR and QSPR (Gordon and Breach, Amsterdam, 1999).

[4] Diudea, M.V. and Gutman, I. Croat. Chem. Acta 71, 21-51, 1998.

[5] Klavžar, S. and Gutman, I. A comparison of the Schultz molecular topological index with the Wiener index, J. Chem. Inf. Comut. Sci. 36, 1001-1003, 1996.

[6] Klein, D.J., Lukovits, I. and Gutman, I. On the definition of the hyper-Wiener index for cycle containing structures, J. Chem. Inf. Comput. Sci. 35, 50-52, 1995.

[7] Lukovits, I. in: Diudea, M.V. (Ed.) QSAR/QSPR studies by molecular descriptors, Nova, Huntigton, 31-38, 2001.

[8] Mirzargar, M. and Ashrafi, A.R. Some distance-based topological indices of a non-commuting graph, Hacettepe J. Math. Stat. 41(4), 515-526, 2012.

[9] Nikolić, S., Trinajstić, N. and Mihalić, Z. The Wiener index: Development and Applications, Croat. Chem. Acta 68, 105-129, 1995.

[10] Randić, M. Novel molecular description for structure property studies, Chem. Phys. Lett. 211, 478-483, 1993.

[11] Rouvray, D.H. Should we have designs on topological indices? In Chemical Applications of Topology and Graph Theory; King, B.B. (Ed. 28 Elsevier, Amsterdam, 1984), 159-177.

[12] Schultz, H.P. Topological organic chemistry 1. Graph theory and topological indices of alkanes, J. Chem. Inf. Comput. Sci. 29, 227-228, 1989.

[13] Wiener, H. Correlation of heats of isomerization and differences in heats of vaporization of isomers, among the paraffin hydrocarbons, J. Am. Chem. Soc. 69, 17-20, 1947.

[14] Wong, G.K. and Coppersmith, D.A. A combinatorial problem related to multimodule memory organization, J. Assoc. Comp. Mach. 21, 392-401, 1974. 